\title{
Local Boundary Feedback Stabilization of the Navier-Stokes Equations
}

\author{
Jean-Pierre Raymond* \\ Université Paul Sabatier \\ Laboratoire MIP, UMR CNRS 5640 \\ 31062 Toulouse Cedex 9, France \\ E-mail: raymondemip.ups-tlse.fr
}

\begin{abstract}
We study the exponential stabilization of the linearized Navier-Stokes equations around an unstable stationary solution, by means of a feedback boundary control, in dimension 2 or 3 . The feedback law is determined by solving a Linear-Quadratic control problem. We do not assume that the normal component of the control is equal to zero. In that case the state equation, satisfied by the velocity field $\mathbf{y}$, is decoupled into an evolution equation satisfied by $P \mathbf{y}$, where $P$ is the so-called Helmholtz projection operator, and a quasi-stationary elliptic equation satisfied by $(I-P) \mathbf{y}$. Using this decomposition we show that the feedback law can be expressed only in function of $P \mathbf{y}$. We show that the linear feedback law provides a local exponential stabilization of the Navier-Stokes equations.
\end{abstract}

Control Systems: Theory, Numerics and Applications

30 March - 1 April 2005

Rome

${ }^{*}$ Speaker. 


\section{Introduction}

Let $\Omega$ be a bounded and connected domain in $\mathbb{R}^{2}$ or $\mathbb{R}^{3}$ with a boundary $\Gamma$ of class $C^{4}, v>0$, and consider a couple $(\mathbf{w}, \chi)$ - a velocity field and a pressure - solution to the stationary NavierStokes equations in $\Omega$ :

$$
-v \Delta \mathbf{w}+(\mathbf{w} \cdot \nabla) \mathbf{w}+\nabla \chi=\mathbf{f} \quad \text { and } \operatorname{div} \mathbf{w}=0 \text { in } \Omega, \quad \mathbf{w}=\mathbf{u}_{s}^{\infty} \quad \text { on } \Gamma .
$$

We assume that $\mathbf{w}$ is regular and is an unstable solution of the instationary Navier-Stokes equations. We want to determine a Dirichlet boundary control $\mathbf{u}$, in feedback form, localized in a part of the boundary $\Gamma$, so that the corresponding controlled system:

$$
\begin{aligned}
& \frac{\partial \mathbf{y}}{\partial t}-v \Delta \mathbf{y}+(\mathbf{y} \cdot \nabla) \mathbf{w}+(\mathbf{w} \cdot \nabla) \mathbf{y}+(\mathbf{y} \cdot \nabla) \mathbf{y}+\nabla p=0, \\
& \operatorname{div} \mathbf{y}=0 \quad \text { in } Q_{\infty}, \quad \mathbf{y}=M \mathbf{u} \text { on } \Sigma_{\infty}, \quad \mathbf{y}(0)=\mathbf{y}_{0} \text { in } \Omega,
\end{aligned}
$$

be stable for initial values $\mathbf{y}_{0}$ small enough in an appropriate space $\mathbf{X}(\Omega)$. In this setting, $Q_{\infty}=$ $\Omega \times(0, \infty), \Sigma_{\infty}=\Gamma \times(0, \infty), \mathbf{X}(\Omega)$ is a subspace of $\mathbf{V}_{n}^{0}(\Omega)=\left\{\mathbf{y} \in \mathbf{L}^{2}(\Omega) \mid \operatorname{div} \mathbf{y}=0\right.$ in $\Omega, \mathbf{y} \cdot \mathbf{n}=$ 0 on $\Gamma\}, \mathbf{w} \in \mathbf{X}(\Omega)$, and the operator $M$ is a restriction operator ensuring that the control is localized on a part of the boundary $\Gamma$ (see [14]). If we set $(\mathbf{z}, q)=(\mathbf{w}+\mathbf{y}, \chi+p)$ and if $\mathbf{u}=0$, we see that $(\mathbf{z}, q)$ is the solution to the Navier-Stokes equations

$$
\begin{aligned}
& \frac{\partial \mathbf{z}}{\partial t}-v \Delta \mathbf{z}+(\mathbf{z} \cdot \nabla) \mathbf{z}+\nabla q=\mathbf{f}, \quad \operatorname{div} \mathbf{z}=0 \quad \text { in } Q_{\infty}, \\
& \mathbf{z}=\mathbf{u}_{s}^{\infty} \text { on } \Sigma_{\infty}, \quad \mathbf{z}(0)=\mathbf{w}+\mathbf{y}_{0} \text { in } \Omega .
\end{aligned}
$$

Thus $\mathbf{y}_{0}$ is a perturbation of the stationary solution w. When $\mathbf{w} \in \mathbf{L}^{\infty}(\Omega)$ and $\mathbf{y}_{0} \in \mathbf{V}_{n}^{0}(\Omega) \cap \mathbf{L}^{4}(\Omega)$ with $\left|\mathbf{y}_{0}\right|_{\mathbf{L}^{4}(\Omega)}$ small enough, the existence of a boundary control $\mathbf{u}$ such that the solution to equation (1.1) exponentially decreases in the norm of the space $\mathbf{X}(\Omega)=\mathbf{V}_{n}^{0}(\Omega) \cap \mathbf{L}^{4}(\Omega)$, follows from a local exact controllability result stated in [5, Theorem 2]. But the proof in [5] does not give any way to define such a control in feedback form. In the three-dimensional case, and when $\mathbf{X}(\Omega)=\{\mathbf{y} \in$ $\mathbf{H}^{1}(\Omega) \mid \operatorname{div} \mathbf{y}=0$ in $\left.\Omega,\langle\mathbf{y} \cdot \mathbf{n}, 1\rangle_{H^{-1 / 2}(\Gamma), H^{1 / 2}(\Gamma)}=0\right\}$, the existence of a control exponentially stabilizing (1.1) is proved in [8]. One way to construct robust feedback laws consists in using the methods of the optimal control theory. This approach has been studied in the case of an internal control $[2,1,3]$, and has been numerically tested with a boundary control in the very specific geometry of the rectangular driven cavity [10] and when the normal component of the control is equal to zero. In many engineering applications $[13,11]$ the normal component of the control variable is not equal to zero. This is the situation we consider here.

The Linear-Quadratic theory for the Dirichlet control of the linearized Navier-Stokes equations has been studied in a very recent work [4], in the case when the normal component of the boundary control is zero, and when the control is applied everywhere on the boundary. To the best of our knowledge the case when the normal component is not equal to zero has not yet been studied in the literature. Our main objectives are:

- first to develop the Linear-Quadratic theory over an infinite time horizon of the Dirichlet boundary control of the Oseen equations when the control is localized on a part of the boundary, and when the normal component of the control is not zero, 
- next to show that the linear feedback law, calculated with the linearized model, and applied to the nonlinear equation (1.1), provides a local exponential stabilization of the state in some appropriate space $\mathbf{X}(\Omega)$.

In the two dimensional case the feedback control law is obtained by studying the control problem

$$
\inf \left\{J(\mathbf{y}, \mathbf{u}) \mid(\mathbf{y}, \mathbf{u}) \text { satisfies }(1.2), \mathbf{u} \in L^{2}\left(0, \infty ; \mathbf{V}^{0}(\Gamma)\right)\right\}
$$

where

and

$$
J(\mathbf{y}, \mathbf{u})=\frac{1}{2} \int_{0}^{\infty} \int_{\Omega}|\mathbf{y}|^{2} d x d t+\frac{1}{2} \int_{0}^{\infty} \int_{\Gamma}|\mathbf{u}|^{2} d x d t
$$

$$
\begin{array}{lll}
\frac{\partial \mathbf{y}}{\partial t}-v \Delta \mathbf{y}+(\mathbf{w} \cdot \nabla) \mathbf{y}+(\mathbf{y} \cdot \nabla) \mathbf{w}-\omega \mathbf{y}+\nabla p=0, & \text { in } Q_{\infty}, \\
\operatorname{div} \mathbf{y}=0 \quad \text { in } Q_{\infty}, \quad \mathbf{y}=M \mathbf{u} \text { on } \Sigma_{\infty}, & \mathbf{y}(0)=\mathbf{y}_{0} & \text { in } \Omega,
\end{array}
$$

where $\mathbf{V}^{0}(\Gamma)=\left\{\mathbf{y} \in \mathbf{L}^{2}(\Gamma) \mid\langle\mathbf{y} \cdot \mathbf{n}, 1\rangle_{H^{-1 / 2}(\Gamma), H^{1 / 2}(\Gamma)}=0\right\}$. The coefficient $\omega>0$, which is not present in (1.1), is added in equation (1.2) in order to guarantee the exponential decay in the norm $\mathbf{H}^{1 / 2-\varepsilon}(\Omega), 0<\varepsilon<1 / 4$, of the solution of the nonlinear closed loop system defined below. We show that the control problem $(\mathscr{P})$ can be rewritten in the form of another control problem in which the state variable is $P \mathbf{y}$ - where $P$ is the so-called Helmholtz projection operator - and not $\mathbf{y}$. This transformation is essential in our approach. It leads to a Riccati equation which is the natural one for the new control problem, but which is not the expected one if we only consider problem $(\mathscr{P})$. This transformation of $(\mathscr{P})$ into a new control problem is a direct consequence of rewriting equation (1.2) in the form:

$$
\begin{aligned}
& P \mathbf{y}^{\prime}=A P \mathbf{y}+\omega P \mathbf{y}+B M \mathbf{u}, \quad \mathbf{y}(0)=\mathbf{y}_{0}, \\
& (I-P) \mathbf{y}=(I-P) D_{A} \gamma_{n} M \mathbf{u} .
\end{aligned}
$$

The operator $A$ is the Oseen operator, the control operator is defined by $B=\left(\lambda_{0} I-A\right) D_{A}$ for some $\lambda_{0}>0$, and $D_{A}$ is the Dirichlet operator associated with $\lambda_{0} I-A$. We refer to [14] for the transformation of equation (1.2) into (1.3), and for regularity results for equation (1.3). Denoting by $\Pi_{\omega}$ the solution to the Riccati equation of the control problem $(\mathscr{P})$, and setting $R_{A}=M D_{A}^{*}(I-$ P) $D_{A} M+I$, we show that the closed loop system

$$
\begin{aligned}
& \frac{\partial \mathbf{y}}{\partial t}-v \Delta \mathbf{y}+(\mathbf{w} \cdot \nabla) \mathbf{y}+(\mathbf{y} \cdot \nabla) \mathbf{w}+\nabla p=0, \quad \text { in } Q_{\infty}, \\
& \operatorname{div} \mathbf{y}=0 \quad \text { in } Q_{\infty}, \quad \mathbf{y}=-M R_{A}^{-1} M B^{*} \Pi_{\omega} P \mathbf{y} \quad \text { on } \Sigma_{\infty}, \quad \mathbf{y}(0)=\mathbf{y}_{0} \quad \text { in } \Omega,
\end{aligned}
$$

is exponentially stable if $\left|\mathbf{y}_{0}\right|_{\mathbf{H}^{1 / 2-\varepsilon}(\Omega) \cap \mathbf{V}_{n}^{0}(\Omega)}$ is small enough for some $0<\varepsilon<1 / 4$.

In the three dimensional case we obtain a similar result by studying the control problem

$$
\inf \left\{I(\mathbf{y}, \mathbf{u}) \mid(\mathbf{y}, \mathbf{u}) \text { satisfies }(1.4), \mathbf{u} \in L^{2}\left(0, \infty ; \mathbf{V}^{0}(\Gamma)\right)\right\}
$$

where

and

$$
I(\mathbf{y}, \mathbf{u})=\frac{1}{2} \int_{0}^{T} \int_{\Omega}\left|(-P \Delta)^{-1 / 2} P \mathbf{y}\right|^{2}+\frac{1}{2} \int_{0}^{T} \int_{\Gamma}\left|R_{A}^{1 / 2} \mathbf{u}\right|^{2}
$$




$$
\begin{aligned}
& \frac{\partial \mathbf{y}}{\partial t}-v \Delta \mathbf{y}+(\mathbf{w} \cdot \nabla) \mathbf{y}+(\mathbf{y} \cdot \nabla) \mathbf{w}-\omega \mathbf{y}+\nabla p=0, \quad \text { in } Q_{\infty}, \\
& \operatorname{div} \mathbf{y}=0 \quad \text { in } Q_{\infty}, \quad \mathbf{y}=\theta(t) M \mathbf{u} \text { on } \Sigma_{\infty}, \quad \mathbf{y}(0)=\mathbf{y}_{0} \quad \text { in } \Omega .
\end{aligned}
$$

The weight function $\theta$ is a $C^{2}$ function from $\mathbb{R}^{+}$into [0,1], satisfying $\theta(0)=0, \theta(t)=1$ for $t \geq T$ for some $T>0$. In that case the solution $\Pi_{\omega}$ of the Riccati equation of the control problem (QQ) depends on $t$ in the intervall $[0, T]$. The exponential decay is obtained in the norm $\mathbf{H}^{1 / 2+\varepsilon}(\Omega)$ if $\left|\mathbf{y}_{0}\right|_{\mathbf{H}_{0}^{1 / 2+\varepsilon}(\Omega) \cap \mathbf{V}_{n}^{0}(\Omega)}$ is small enough.

To simplify the presentation, throughout the paper we assume that $v=1$ and $M=I$. For the extension of results to the case when $M \neq I$ we refer to [16] and [15]. ( $M=I$ corresponds to the case where $\mathbf{u}$ acts everywhere on $\Gamma$.)

\section{Oseen equation}

Let us introduce the following function spaces : $H^{s}\left(\Omega ; \mathbb{R}^{N}\right)=\mathbf{H}^{s}(\Omega), L^{2}\left(\Omega ; \mathbb{R}^{N}\right)=\mathbf{L}^{2}(\Omega)$, the same notation conventions are used for the spaces $H_{0}^{s}\left(\Omega ; \mathbb{R}^{N}\right)$, and the trace spaces $H^{s}\left(\Gamma ; \mathbb{R}^{N}\right)$. Throughout what follows, for all $\mathbf{u} \in \mathbf{L}^{2}(\Omega)$ such that $\operatorname{div} \mathbf{u} \in L^{2}(\Omega)$, we denote by $\mathbf{u} \cdot \mathbf{n}$ the normal trace of $\mathbf{u}$ in $H^{-1 / 2}(\Gamma)$ [17]. Following [7], we use the letter $\mathbf{V}$ to define different spaces of divergence free vector functions and for some associated trace spaces:

$$
\begin{aligned}
& \mathbf{V}^{s}(\Omega)=\left\{\mathbf{u} \in \mathbf{H}^{s}(\Omega) \mid \operatorname{div} \mathbf{u}=0 \text { in } \Omega,\langle\mathbf{u} \cdot \mathbf{n}, 1\rangle_{H^{-1 / 2}(\Gamma), H^{1 / 2}(\Gamma)}=0\right\} \quad \text { for } s \geq 0, \\
& \mathbf{V}_{n}^{s}(\Omega)=\left\{\mathbf{u} \in \mathbf{H}^{s}(\Omega) \mid \operatorname{div} \mathbf{u}=0 \text { in } \Omega, \mathbf{u} \cdot \mathbf{n}=0 \text { on } \Gamma\right\} \quad \text { for } s \geq 0, \\
& \mathbf{V}_{0}^{s}(\Omega)=\left\{\mathbf{u} \in \mathbf{H}^{s}(\Omega) \mid \operatorname{div} \mathbf{u}=0 \text { in } \Omega, \mathbf{u}=0 \text { on } \Gamma\right\} \quad \text { for } s>1 / 2, \\
& \mathbf{V}^{s}(\Gamma)=\left\{\mathbf{u} \in \mathbf{H}^{s}(\Gamma) \mid \int_{\Gamma} \mathbf{u} \cdot \mathbf{n}=0\right\} \quad \text { for } s \geq 0 .
\end{aligned}
$$

For $s<0, \mathbf{V}^{s}(\Gamma)$ is the dual space of $\mathbf{V}^{-s}(\Gamma)$, with $\mathbf{V}^{0}(\Gamma)$ as pivot space. For spaces of time dependent functions we set

$$
\mathbf{V}^{s, \sigma}(Q)=H^{\sigma}\left(0, T ; \mathbf{V}^{0}(\Omega)\right) \cap L^{2}\left(0, T ; \mathbf{V}^{s}(\Omega)\right),
$$

and

$$
\mathbf{V}^{s, \sigma}(\Sigma)=H^{\sigma}\left(0, T ; \mathbf{V}^{0}(\Gamma)\right) \cap L^{2}\left(0, T ; \mathbf{V}^{s}(\Gamma)\right)
$$

Observe that

$$
\mathbf{V}^{s, \sigma}(Q)=\mathbf{H}^{s, \sigma}(Q) \cap L^{2}\left(0, T ; \mathbf{V}^{0}(\Omega)\right) \quad \text { for all } s \geq 0 \text { and } \sigma \geq 0,
$$

where $\mathbf{H}^{s, \sigma}(Q)=\left(H^{s, \sigma}(Q)\right)^{N}$, and $H^{s, \sigma}(Q)$ corresponds to the notation in [12].

We denote by $\gamma_{\tau} \in \mathscr{L}\left(\mathbf{V}^{0}(\Gamma)\right)$ and $\gamma_{n} \in \mathscr{L}\left(\mathbf{V}^{0}(\Gamma)\right)$ the operators defined by

$$
\gamma_{n} \mathbf{u}=(\mathbf{u} \cdot \mathbf{n}) \mathbf{n} \quad \text { and } \quad \gamma_{\tau} \mathbf{u}=\mathbf{u}-\gamma_{n} \mathbf{u} \quad \text { for all } \mathbf{u} \in \mathbf{V}^{0}(\Gamma) .
$$

As usual, for $s>1 / 2, \gamma_{0} \in \mathscr{L}\left(\mathbf{V}^{s}(\Omega), \mathbf{V}^{s-1 / 2}(\Gamma)\right)$ denotes the trace operator. 
Let us denote by $P$ the orthogonal projection operator in $\mathbf{L}^{2}(\Omega)$ on $\mathbf{V}_{n}^{0}(\Omega)$. Recall that the Stokes operator $A_{0}=P \Delta$, with domain $D\left(A_{0}\right)=\mathbf{V}^{2}(\Omega) \cap \mathbf{V}_{0}^{1}(\Omega)$ in $\mathbf{V}_{n}^{0}(\Omega)$, is the infinitesimal generator of a strongly continuous analytic semigroup $\left(e^{t A_{0}}\right)_{t>0}$ on $\mathbf{V}_{n}^{0}(\Omega)$.

To study the stabilization of the Navier-Stokes equations around the unstable stationary solution $\mathbf{w}$, we consider the Oseen equation

$$
\begin{aligned}
& \frac{\partial \mathbf{y}}{\partial t}-\Delta \mathbf{y}+(\mathbf{w} \cdot \nabla) \mathbf{y}+(\mathbf{y} \cdot \nabla) \mathbf{w}+\nabla p=0, \operatorname{div} \mathbf{y}=0 \quad \text { in } Q, \\
& \mathbf{y}=\mathbf{u} \text { on } \Sigma, \quad \mathbf{y}(0)=\mathbf{y}_{0} \text { in } \Omega,
\end{aligned}
$$

where $\mathbf{w}$ belongs to $\mathbf{V}^{3}(\Omega)$.

To study equation (2.1) we introduce the unbounded operators $A$ and $A^{*}$ in $\mathbf{V}_{n}^{0}(\Omega)$ defined by

$$
\begin{aligned}
& D(A)=\left\{\mathbf{y} \in \mathbf{V}_{0}^{1}(\Omega) \mid P \Delta \mathbf{y}-P((\mathbf{w} \cdot \nabla) \mathbf{y})-P((\mathbf{y} \cdot \nabla) \mathbf{w}) \in \mathbf{V}_{n}^{0}(\Omega)\right\}=\mathbf{V}^{2}(\Omega) \cap \mathbf{V}_{0}^{1}(\Omega), \\
& D\left(A^{*}\right)=\left\{\mathbf{y} \in \mathbf{V}_{0}^{1}(\Omega) \mid P \Delta \mathbf{y}+P((\mathbf{w} \cdot \nabla) \mathbf{y})-P\left((\nabla \mathbf{w})^{T} \mathbf{y}\right) \in \mathbf{V}_{n}^{0}(\Omega)\right\}=\mathbf{V}^{2}(\Omega) \cap \mathbf{V}_{0}^{1}(\Omega), \\
& A \mathbf{y}=P \Delta \mathbf{y}-P((\mathbf{w} \cdot \nabla) \mathbf{y})-P((\mathbf{y} \cdot \nabla) \mathbf{w}) \quad \text { and } \quad A^{*} \mathbf{y}=P \Delta \mathbf{y}+P((\mathbf{w} \cdot \nabla) \mathbf{y})-P\left((\nabla \mathbf{w})^{T} \mathbf{y}\right) .
\end{aligned}
$$

Throughout what follows we assume that $\lambda_{0}>0$ is such that

and

$$
\int_{\Omega}\left(\lambda_{0}|\mathbf{y}|^{2}+|\nabla \mathbf{y}|^{2}+((\mathbf{w} \cdot \nabla) \mathbf{y}) \cdot \mathbf{y}+((\mathbf{y} \cdot \nabla) \mathbf{w}) \cdot \mathbf{y}\right) d x \geq \frac{1}{2} \int_{\Omega}\left(|\mathbf{y}|^{2}+|\nabla \mathbf{y}|^{2}\right) d x
$$

$$
\left.\int_{\Omega}\left(\lambda_{0}|\mathbf{y}|^{2}+|\nabla \mathbf{y}|^{2}-((\mathbf{w} \cdot \nabla) \mathbf{y}) \cdot \mathbf{y}+((\nabla) \mathbf{w})^{T} \mathbf{y}\right) \cdot \mathbf{y}\right) d x \geq \frac{1}{2} \int_{\Omega}\left(|\mathbf{y}|^{2}+|\nabla \mathbf{y}|^{2}\right) d x
$$

for all $\mathbf{y} \in \mathbf{V}_{0}^{1}(\Omega)$.

The semigroup $\left(e^{t A}\right)_{t \geq 0}$ on $\mathbf{V}_{n}^{0}(\Omega)$, generated by $(A, D(A))$, can be continuously extended to $\left(D\left(A^{*}\right)\right)^{\prime}=\left(\mathbf{V}^{2}(\Omega) \cap \mathbf{V}_{0}^{1}(\Omega)\right)^{\prime}$. This corresponds to the so-called extrapolation method. We shall denote by $\left(e^{t \tilde{A}}\right)_{t \geq 0}$ the extended semigroup, and by $(\tilde{A}, D(\tilde{A}))$ its infinitesimal generator. It is well known that $D(\tilde{A})=\mathbf{V}_{n}^{0}(\Omega)$.

To rewrite equation (2.1) in the form of an evolution equation, we introduce the Dirichlet operators $D_{A}$ and $D_{p, A}$ associated with $\lambda_{0} I-A$, defined by $\left(D_{A} \mathbf{g}, D_{p, A} \mathbf{g}\right)=(\mathbf{v}, \pi)$, where, for all $\mathbf{g} \in \mathbf{V}^{0}(\Gamma),(\mathbf{v}, \pi)$ is the solution to the equation

$$
\lambda_{0} \mathbf{v}-\Delta \mathbf{v}+(\mathbf{w} \cdot \nabla) \mathbf{v}+(\mathbf{v} \cdot \nabla) \mathbf{w}+\nabla \pi=0 \quad \text { and } \quad \operatorname{div} \mathbf{v}=0 \text { in } \Omega, \quad \mathbf{v}=\mathbf{g} \quad \text { on } \Gamma .
$$

Definition 1. A function $\mathbf{y} \in L^{2}\left(0, T ; \mathbf{V}^{0}(\Omega)\right)$ is a weak solution to equation (2.1) if

$P \mathbf{y}$ is a weak solution of the evolution equation

$$
\begin{gathered}
P \mathbf{y}^{\prime}=\tilde{A} P \mathbf{y}+\left(\lambda_{0} I-\tilde{A}\right) P D_{A} \mathbf{u}, \quad P \mathbf{y}(0)=P \mathbf{y}_{0}, \\
\text { and } \quad \\
(I-P) \mathbf{y}(\cdot)=(I-P) D_{A} \gamma_{n} \mathbf{u}(\cdot) \quad \text { in } L^{2}\left(0, T ; \mathbf{V}^{0}(\Omega)\right) .
\end{gathered}
$$

An alternative definition can be stated by using $\left(e^{t \tilde{A}_{0}}\right)_{t \geq 0}$ and its infinitesimal generator $\left(\tilde{A}_{0}\right.$, $\left.D\left(\tilde{A}_{0}\right)\right)$, where $\left(e^{t \tilde{A}_{0}}\right)_{t \geq 0}$ is the extension to $\left(D\left(A_{0}^{*}\right)\right)^{\prime}=\left(\mathbf{V}^{2}(\Omega) \cap \mathbf{V}_{0}^{1}(\Omega)\right)^{\prime}$ of the Stokes semigroup 
$\left(e^{t A_{0}}\right)_{t \geq 0}$. More precisely, it can be shown (see [14, Theorem 4.2]) that $\mathbf{y} \in L^{2}\left(0, T ; \mathbf{V}^{0}(\Omega)\right.$ ) is a weak solution to equation (2.1) in the sense of Definition 1 if and only if

$P \mathbf{y}$ is a weak solution of the evolution equation

$$
P \mathbf{y}^{\prime}=\tilde{A}_{0} P \mathbf{y}+\left(-\tilde{A}_{0}\right) P D \mathbf{u}-P(\operatorname{div}(\mathbf{w} \otimes \mathbf{y}))-P(\operatorname{div}(\mathbf{y} \otimes \mathbf{w})), \quad P \mathbf{y}(0)=P \mathbf{y}_{0},
$$

and

$$
(I-P) \mathbf{y}(\cdot)=(I-P) D \mathbf{u}(\cdot)=(I-P) D \gamma_{n} \mathbf{u}(\cdot),
$$

where $D \mathbf{g}=\mathbf{v}$ is the solution to the equation

$$
-\Delta \mathbf{v}+\nabla \pi=0 \quad \text { and } \quad \operatorname{div} \mathbf{v}=0 \text { in } \Omega, \quad \mathbf{v}=\mathbf{g} \quad \text { on } \Gamma .
$$

We can state the following regularity result.

Theorem 1. ([14,Theorem 4.1]) (i) For all $P \mathbf{y}_{0} \in \mathbf{V}_{n}^{0}(\Omega)$ and all $\mathbf{u} \in L^{2}\left(0, T ; \mathbf{V}^{0}(\Gamma)\right)$ equation (2.1), admits a unique weak solution in $L^{2}\left(0, T ; \mathbf{V}^{0}(\Omega)\right)$ in the sense of Definition 1. This solution obeys

$$
\begin{aligned}
& \|P \mathbf{y}\|_{L^{2}\left(0, T ; \mathbf{V}_{n}^{1 / 2-\varepsilon}(\Omega)\right)}+\|P \mathbf{y}\|_{H^{1 / 4-\varepsilon / 2}\left(0, T ; \mathbf{V}_{n}^{0}(\Omega)\right)}+\|(I-P) \mathbf{y}\|_{L^{2}\left(0, T ; \mathbf{V}^{1 / 2}(\Omega)\right)} \\
& \leq C\left(\left\|P \mathbf{y}_{0}\right\|_{\mathbf{V}_{n}^{0}(\Omega)}+\|\mathbf{u}\|_{L^{2}\left(0, T ; \mathbf{V}^{0}(\Gamma)\right)}\right) \quad \text { for all } \varepsilon>0 .
\end{aligned}
$$

(ii) If $\mathbf{u} \in \mathbf{V}^{s, s / 2}(\Sigma)$ with $0 \leq s \leq 2$, then

$$
\|(I-P) \mathbf{y}\|_{L^{2}\left(0, T ; \mathbf{V}^{s+1 / 2}(\Omega)\right)}+\|(I-P) \mathbf{y}\|_{H^{s / 2}\left(0, T ; \mathbf{V}^{1 / 2}(\Omega)\right)} \leq C\|\mathbf{u}\|_{\mathbf{V}^{s, s}(\Sigma)} .
$$

(iii) If $\mathbf{u} \in \mathbf{V}^{s, s / 2}(\Sigma)$ and $P \mathbf{y}_{0} \in \mathbf{V}_{n}^{0 \wedge(s-1 / 2)}(\Omega)$, with $0 \leq s<1$, then

$$
\|P \mathbf{y}\|_{\mathbf{V}^{s+1 / 2-\varepsilon, s / 2+1 / 4-\varepsilon / 2}(Q)} \leq C\left(\left\|P \mathbf{y}_{0}\right\|_{\mathbf{V}_{n}^{0 \wedge(s-1 / 2)}(\Omega)}+\|\mathbf{u}\|_{\mathbf{V}^{s, s / 2}(\Sigma)}\right) \quad \text { for all } \varepsilon>0 .
$$

(iv) If $\mathbf{u} \in \mathbf{V}^{s, s / 2}(\Sigma), P \mathbf{y}_{0} \in \mathbf{V}_{n}^{s-1 / 2}(\Omega)$, with $1<s \leq 2$, and if $P \mathbf{y}_{0}$ and $\mathbf{u}(0)$ satisfy the compatibility condition

$$
\gamma_{0}\left(P\left(\mathbf{y}_{0}-D \mathbf{u}(0)\right)\right)=0
$$

then the estimate (2.3) is satisfied.

\section{A finite time horizon problem}

To deal with the stabilization problem formulated in the introduction, we first study the following optimal control problem

$$
\inf \left\{J_{T}(\mathbf{y}, \mathbf{u}) \mid(\mathbf{y}, \mathbf{u}) \text { satisfies }(3.1), \mathbf{u} \in \mathbf{V}^{0,0}\left(\Sigma_{0, T}\right)\right\},
$$

where

$$
J_{T}(\mathbf{y}, \mathbf{u})=\frac{1}{2} \int_{0}^{T} \int_{\Omega}|\mathbf{y}|^{2} d x d t+\frac{1}{2} \int_{0}^{T}|\mathbf{u}(t)|_{\mathbf{V}^{0}(\Gamma)}^{2} d t
$$

and

$$
\begin{aligned}
& \frac{\partial \mathbf{y}}{\partial t}-\Delta \mathbf{y}+(\mathbf{w} \cdot \nabla) \mathbf{y}+(\mathbf{y} \cdot \nabla) \mathbf{w}+\nabla p=0 \quad \text { in } Q_{0, T}=\Omega \times(0, T) \\
& \operatorname{div} \mathbf{y}=0 \quad \text { in } Q_{0, T}, \quad \mathbf{y}=\mathbf{u} \text { on } \Sigma_{0, T}=\Gamma \times(0, T), \quad \mathbf{y}(0)=\zeta \quad \text { in } \Omega .
\end{aligned}
$$


Thus, if $\mathbf{y}$ is the solution of (3.1), we have

$$
J_{T}(\mathbf{y}, \mathbf{u})=I_{T}(\mathbf{y}, \mathbf{u})=\frac{1}{2} \int_{0}^{T} \int_{\Omega}|P \mathbf{y}|^{2}+\frac{1}{2} \int_{0}^{T} \int_{\Gamma}\left|R_{A}^{1 / 2} \gamma_{n} \mathbf{u}\right|^{2}+\frac{1}{2} \int_{0}^{T} \int_{\Gamma}\left|\gamma_{\tau} \mathbf{u}\right|^{2},
$$

where $R_{A}$ is the operator defined by

$$
R_{A}=D_{A}^{*}(I-P) D_{A}+I
$$

The control problem $\left(\mathscr{Q}_{\zeta}^{T}\right)$ is equivalent to

$\left(\mathscr{P}_{\zeta}^{T}\right) \quad \inf \left\{I_{T}(\mathbf{y}, \mathbf{u}) \mid(\mathbf{y}, \mathbf{u})\right.$ satisfies $\left.(2.3), \mathbf{u} \in \mathbf{V}^{0,0}\left(\Sigma_{0, T}\right)\right\}$,

where

$$
P \mathbf{y}^{\prime}=A P \mathbf{y}+B \mathbf{u} \quad \text { in }(0, T), \quad P \mathbf{y}(0)=\zeta, \quad \text { and } \quad(I-P) \mathbf{y}=(I-P) D_{A} \gamma_{n} \mathbf{u}
$$

with $B=\left(\lambda_{0} I-A\right) P D_{A}$.

Problem $\left(\mathscr{P}_{\zeta}^{T}\right)$ admits a unique solution $\left(\mathbf{y}_{\zeta}, \mathbf{u}_{\zeta}\right)$, where

$$
\mathbf{u}_{\zeta}=-R_{A}^{-1} B^{*} \Phi_{\zeta}
$$

and $\left(\mathbf{y}_{\zeta}, \Phi_{\zeta}\right)$ is the unique solution to the system

$$
\begin{aligned}
& P \mathbf{y}^{\prime}=A P \mathbf{y}-B R_{A}^{-1} B^{*} \Phi \quad \text { in }(0, T), \quad P \mathbf{y}(0)=\zeta, \quad(I-P) \mathbf{y}=-(I-P) D_{A} \gamma_{n} R_{A}^{-1} B^{*} \Phi, \\
& -\Phi^{\prime}=A^{*} \Phi+P \mathbf{y} \quad \text { in }(0, T), \quad \Phi(T)=0 .
\end{aligned}
$$

The operator $B^{*}$ is determined by

$$
B^{*} \Phi=-\frac{\partial \Phi}{\partial \mathbf{n}}+\psi \mathbf{n}-\frac{1}{|\Gamma|} \int_{\Gamma} \psi \mathbf{n}, \quad \forall \Phi \in \mathbf{V}^{2}(\Omega) \cap \mathbf{V}_{0}^{1}(\Omega)
$$

where $|\Gamma|$ is the $(N-1)$-dimensional Lebesgue measure of $\Gamma$, and $\psi \in H^{1}(\Omega) / \mathbb{R}$ is the unique solution of

$$
\nabla \psi=(I-P)\left[\Delta \Phi+(\mathbf{w} \cdot \nabla) \Phi-(\nabla \mathbf{w})^{T} \Phi\right]
$$

If we denote by $\Pi(T) \in \mathscr{L}\left(\mathbf{V}_{n}^{0}(\Omega)\right)$ the mapping

$$
\Pi(T): \zeta \longmapsto \Phi_{\zeta}(0)
$$

we can prove that $\Pi(T)=\Pi(T)^{*} \geq 0$, and that it also belongs to $\mathscr{L}\left(\mathbf{V}_{n}^{0}(\Omega), \mathbf{V}^{2}(\Omega) \cap \mathbf{V}_{0}^{1}(\Omega)\right)$. Moreover the value function of $\left(\mathscr{P}_{\zeta}^{T}\right)$ and $\Pi(T)$ obeys the identity:

$$
\inf \left(\mathscr{P}_{\zeta}^{T}\right)=\frac{1}{2}(\Pi(T) \zeta, \zeta)_{\mathbf{V}_{n}^{0}(\Omega)} .
$$




\section{An infinite time horizon problem}

Now we want to study the problem $\left(\mathscr{P}_{\zeta}^{\infty}\right)$, and we want to study the regularity of its solution in function of the regularity of $\zeta$. Let us recall that problem $\left(\mathscr{P}_{\zeta}^{\infty}\right)$ is defined by

$$
\inf \left\{I(\mathbf{y}, \mathbf{u}) \mid(\mathbf{y}, \mathbf{u}) \text { satisfies }(4.1), \mathbf{u} \in \mathbf{V}^{0,0}\left(\Sigma_{\infty}\right)\right\},
$$

where

$$
I(\mathbf{y}, \mathbf{u})=\frac{1}{2} \int_{0}^{\infty} \int_{\Omega}|P \mathbf{y}|^{2} d x d t+\frac{1}{2} \int_{0}^{\infty}\left(\left|\gamma_{\tau} \mathbf{u}(t)\right|_{\mathbf{V}^{0}(\Gamma)}^{2}+\left|R_{A}^{1 / 2} \gamma_{n} \mathbf{u}(t)\right|_{\mathbf{V}^{0}(\Gamma)}^{2}\right) d t
$$

and

$$
P \mathbf{y}^{\prime}=A P \mathbf{y}+B \mathbf{u} \quad \text { in }(0, \infty), \quad \mathbf{y}(0)=\zeta, \quad \text { and } \quad(I-P) \mathbf{y}=(I-P) D_{A} \gamma_{n} \mathbf{u} .
$$

Using the null controllability result stated in [5], we can show that there exists a control $\mathbf{u} \in$ $\mathbf{V}^{0,0}\left(\Sigma_{\infty}\right)$ such that the corresponding solution $\mathbf{y}_{\mathbf{u}}$ to equation (4.1) obeys

$$
I\left(\mathbf{y}_{\mathbf{u}}, \mathbf{u}\right)<\infty .
$$

Thus, using the so-called direct method, we can prove that problem $\left(\mathscr{P}_{\zeta}^{\infty}\right)$ admits a unique solution $\left(\mathbf{y}_{\zeta}, \mathbf{u}_{\zeta}\right)$, that we want to characterize.

From the dynamic programming principle it follows that, for all $\zeta \in \mathbf{V}_{n}^{0}(\Omega)$, the mapping

$$
T \longmapsto(\Pi(T) \zeta, \zeta)_{\mathbf{V}_{n}^{0}(\Omega)}
$$

is nondecreasing. Moreover we have

$$
(\Pi(T) \zeta, \zeta)_{\mathbf{V}_{n}^{0}(\Omega)} \leq I\left(\mathbf{y}_{\zeta}, \mathbf{u}_{\zeta}\right)<\infty .
$$

Therefore there exists an operator $\Pi \in \mathscr{L}\left(\mathbf{V}_{n}^{0}(\Omega)\right)$ satisfying $\Pi=\Pi^{*}$ and

$$
\Pi \zeta=\lim _{T \rightarrow \infty} \Pi(T) \zeta \quad \text { for all } \zeta \in \mathbf{V}_{n}^{0}(\Omega) .
$$

Thus we have proved the following result.

Theorem 2. For all $\zeta \in \mathbf{V}_{n}^{0}(\Omega)$, problem $\left(\mathscr{P}_{\zeta}^{\infty}\right)$ admits a unique solution $\left(\mathbf{y}_{\zeta}, \mathbf{u}_{\zeta}\right)$. There exists $\Pi \in \mathscr{L}\left(\mathbf{V}_{n}^{0}(\Omega)\right)$, obeying $\Pi=\Pi^{*} \geq 0$, such that the optimal cost is given by

$$
I\left(\mathbf{y}_{\zeta}, \mathbf{u}_{\zeta}\right)=\frac{1}{2}(\Pi \zeta, \zeta)_{\mathbf{V}_{n}^{0}(\Omega)}
$$

To study the regularizing property of the operator $\Pi$, we first establish the following theorem.

Theorem 3. For every $\zeta \in \mathbf{V}_{n}^{0}(\Omega)$, the system

$$
\begin{aligned}
& P \mathbf{y}^{\prime}=A P \mathbf{y}-B R_{A}^{-1} B^{*} \Phi \quad \text { in }(0, \infty), \quad \mathbf{y}(0)=\zeta, \\
& (I-P) \mathbf{y}=-(I-P) D_{A} \gamma_{n} R_{A}^{-1} B^{*} \Phi, \\
& -\Phi^{\prime}=A^{*} \Phi+P \mathbf{y} \quad \text { in }(0, \infty), \quad \Phi(\infty)=0, \\
& \Phi(t)=\Pi P \mathbf{y}(t) \quad \text { for all } t \in(0, \infty),
\end{aligned}
$$


admits a unique solution in $L^{2}\left(0, \infty ; \mathbf{V}^{0}(\Omega)\right) \times \mathbf{V}^{2,1}\left(Q_{\infty}\right)$. This solution satisfies:

$$
\begin{aligned}
& \|(I-P) \mathbf{y}\|_{L^{2}\left(0, \infty ; \mathbf{V}^{3 / 2}(\Omega)\right)}+\|(I-P) \mathbf{y}\|_{H^{1 / 2}\left(0, \infty ; \mathbf{V}^{0}(\Omega)\right)} \\
& +\|P \mathbf{y}\|_{C_{b}\left(\mathbb{R}^{+} ; \mathbf{V}_{n}^{0}(\Omega)\right)}+\|P \mathbf{y}\|_{\mathbf{V}^{1,1 / 2}\left(Q_{\infty}\right)}+\|\Phi\|_{\mathbf{V}^{3,3 / 2}\left(Q_{\infty}\right)} \leq C|\zeta|_{\mathbf{V}_{n}^{0}(\Omega)} .
\end{aligned}
$$

The pair $\left(\mathbf{y},-R_{A}^{-1} B^{*} \Phi\right)$ is the solution of $\left(\mathscr{P}_{\zeta}^{\infty}\right)$.

From Theorem 3 we deduce that $\Phi_{\zeta}$ belongs to $C_{b}\left(\mathbb{R}^{+} ; \mathbf{V}^{2}(\Omega) \cap \mathbf{V}_{0}^{1}(\Omega)\right)$. In particular we have

$$
\left|\Phi_{\zeta}(0)\right|_{\mathbf{V}^{2}(\Omega) \cap \mathbf{V}_{0}^{1}(\Omega)} \leq C|\zeta|_{\mathbf{V}_{n}^{0}(\Omega)} .
$$

This means that $\Pi$ is also a bounded operator from $\mathbf{V}_{n}^{0}(\Omega)$ into $\mathbf{V}^{2}(\Omega) \cap \mathbf{V}_{0}^{1}(\Omega)$.

Now using Theorem 3 , the definition of $B^{*}$, and regularity results for the Oseen equation, we can prove the following regularity result.

Corollary 1. ([15, Corollary 4.3]) If $\zeta \in \mathbf{V}_{n}^{1 / 2-\varepsilon}(\Omega)$ for some $0<\varepsilon<1 / 2$, then the solution $(\mathbf{y}, \Phi)$ of system (4.2) belongs to $\mathbf{V}^{3 / 2-\varepsilon, 3 / 4-\varepsilon / 2}\left(Q_{\infty}\right) \times \mathbf{V}^{7 / 2-\varepsilon, 7 / 4-\varepsilon / 2}\left(Q_{\infty}\right)$, and we have:

$$
\|\mathbf{y}\|_{\mathbf{V}^{3 / 2-\varepsilon, 3 / 4-\varepsilon / 2}\left(Q_{\infty}\right)}+\|\Phi\|_{\mathbf{V}^{7 / 2-\varepsilon, 7 / 4-\varepsilon / 2}\left(Q_{\infty}\right)}+\left\|B^{*} \Phi\right\|_{\mathbf{V}^{3 / 2-\varepsilon, 3 / 4-\varepsilon / 2}\left(Q_{\infty}\right)} \leq C|\zeta|_{\mathbf{V}_{n}^{1 / 2-\varepsilon}(\Omega)} .
$$

From Corollary 1 it follows that $\Pi$ is a bounded operator from $\mathbf{V}_{n}^{1 / 2-\varepsilon}(\Omega)$ into $\mathbf{V}^{5 / 2-\varepsilon}(\Omega) \cap$ $\mathbf{V}_{0}^{1}(\Omega)$, and the operator $B^{*} \Pi$ is bounded from $\mathbf{V}_{n}^{1 / 2-\varepsilon}(\Omega)$ into $\mathbf{V}_{n}^{1-\varepsilon}(\Gamma)$ for all $0<\varepsilon<1 / 2$.

With Theorem 3, we can easily establish that the family of operators

$$
\left(\zeta \longmapsto P \mathbf{y}_{\zeta}(t)\right)_{t \geq 0}
$$

is an exponentially stable semigroup on $\mathbf{V}_{n}^{0}(\Omega)$. Let us denote it by $\left(e^{t A_{\Pi}}\right)_{t \geq 0}$, and let $\left(A_{\Pi}, D\left(A_{\Pi}\right)\right)$ be its infinitesimal generator. For all $\mathbf{f} \in \mathbf{V}_{n}^{0}(\Omega)$ the equation

$$
\mathbf{y} \in \mathbf{V}_{n}^{0}(\Omega), \quad A_{\Pi} \mathbf{y}=\mathbf{f},
$$

admits a unique solution given by

$$
\mathbf{y}=\int_{0}^{\infty} e^{t A_{\Pi}} \mathbf{f} d t
$$

Therefore $\mathbf{y} \in D\left(A_{\Pi}\right)$ if and only if there exists $\mathbf{f} \in \mathbf{V}_{n}^{0}(\Omega)$ such that $\mathbf{y}=\int_{0}^{\infty} e^{t A_{\Pi}} \mathbf{f} d t$. It can also be proved that $\mathbf{y} \in D\left(A_{\Pi}\right)$ if and only if $\mathbf{y} \in \mathbf{V}_{n}^{0}(\Omega)$ and $A \mathbf{y}-B R_{A}^{-1} B^{*} \Pi \mathbf{y} \in \mathbf{V}_{n}^{0}(\Omega)$.

Theorem 4. ([15, Theorem 4.5]) The operator $\Pi$ is the unique weak solution to the algebraic Riccati equation

$$
\begin{aligned}
& \Pi^{*}=\Pi \in \mathscr{L}\left(\mathbf{V}_{n}^{0}(\Omega)\right) \quad \text { and } \quad \Pi \geq 0, \\
& \text { for all } \mathbf{y} \in \mathbf{V}_{n}^{0}(\Omega), \Pi \mathbf{y} \in \mathbf{V}^{2}(\Omega) \cap \mathbf{V}_{0}^{1}(\Omega) \quad \text { and } \quad|\Pi \mathbf{y}|_{\mathbf{V}^{2}(\Omega)} \leq C|\mathbf{y}|_{\mathbf{V}_{n}^{0}(\Omega)}, \\
& A^{*} \Pi+\Pi A-\Pi B_{\tau} B_{\tau}^{*} \Pi-\Pi B_{n} R_{A}^{-1} B_{n}^{*} \Pi+I=0,
\end{aligned}
$$

where $B_{\tau}=B \gamma_{\tau}$ and $B_{n}=B \gamma_{n}$. 


\section{Stabilization of the two dimensional Navier-Stokes equations}

Throughout this section, we assume that $N=2$.

\subsection{First stabilization result}

Consider the Navier-Stokes equations with the linear feedback law determined in the previous section:

$$
\begin{array}{ll}
P \mathbf{y}^{\prime}=A_{\Pi} P \mathbf{y}+F(\mathbf{y}) \quad \text { in }(0, \infty), & P \mathbf{y}(0)=\mathbf{y}_{0}, \\
(I-P) \mathbf{y}=-(I-P) D_{A} R_{A}^{-1} B_{n}^{*} \Pi P \mathbf{y} & \text { in }(0, \infty),
\end{array}
$$

where $F(\mathbf{y})=-P(\mathbf{y} \cdot \nabla) \mathbf{y}$.

We can now state our first stabilization result.

Theorem 5. ([15, Theorem 6.1]) For all $0<\varepsilon<1 / 4$, there exists $\mu_{0}>0$ and a nondecreasing function $\eta$ from $\mathbb{R}^{+}$into itself, such that if $\mu \in\left(0, \mu_{0}\right)$ and $|\zeta|_{\mathbf{V}_{n}^{1 / 2-\varepsilon}(\Omega)} \leq \eta(\mu)$, then equation (5.1) admits a unique solution in the set

$$
D_{\mu}=\left\{\mathbf{y} \in \mathbf{V}^{3 / 2-\varepsilon, 3 / 4-\varepsilon / 2}\left(Q_{\infty}\right) \mid\|\mathbf{y}\|_{\mathbf{V}^{3 / 2-\varepsilon, 3 / 4-\varepsilon / 2}\left(Q_{\infty}\right)} \leq \mu\right\} .
$$

Moreover $(I-P) \mathbf{y}$ belongs to $H^{3 / 4-\varepsilon / 2}\left(0, \infty ; \mathbf{V}^{1 / 2}(\Omega)\right) \cap L^{2}\left(0, \infty ; \mathbf{V}^{3 / 2-\varepsilon}(\Omega)\right)$.

To prove this theorem, we first show that the mapping

$$
\mathbf{z} \longmapsto F(\mathbf{z})
$$

is locally Lipschitz from $\mathbf{V}^{3 / 2-\varepsilon, 3 / 4-\varepsilon / 2}\left(Q_{\infty}\right)$ into $L^{2 /(1+2 \varepsilon)}\left(0, \infty ; \mathbf{V}_{n}^{0}(\Omega)\right) \cap L^{2}\left(0, \infty ;\left(\mathbf{V}^{2 \varepsilon}(\Omega)\right)^{\prime}\right)$. More precisely we have

$$
\|P F(\mathbf{z})\|_{L^{2 /(1+2 \varepsilon)}\left(0, \infty ; \mathbf{V}_{n}^{0}(\Omega)\right)}+\|P F(\mathbf{z})\|_{L^{2}\left(0, \infty ;\left(\mathbf{V}^{2 \varepsilon}(\Omega)\right)^{\prime}\right)} \leq C\|\mathbf{z}\|_{\mathbf{V}^{3 / 2-\varepsilon, 3 / 4-\varepsilon / 2}\left(Q_{\infty}\right)}^{2},
$$

and

$$
\begin{aligned}
& \left\|P F\left(\mathbf{z}_{1}\right)-P F\left(\mathbf{z}_{2}\right)\right\|_{L^{2 /(1+2 \varepsilon)}\left(0, \infty ; \mathbf{V}_{n}^{0}(\Omega)\right)}+\left\|P F\left(\mathbf{z}_{1}\right)-P F\left(\mathbf{z}_{2}\right)\right\|_{L^{2}\left(0, \infty ;\left(\mathbf{V}^{2 \varepsilon}(\Omega)\right)^{\prime}\right)} \\
& \leq C\left(\left\|\mathbf{z}_{1}\right\|_{\mathbf{V}^{3 / 2-\varepsilon, 3 / 4-\varepsilon / 2}\left(Q_{\infty}\right)}+\left\|\mathbf{z}_{2}\right\|_{\mathbf{V}^{3 / 2-\varepsilon, 3 / 4-\varepsilon / 2}\left(Q_{\infty}\right)}\right)\left\|\mathbf{z}_{1}-\mathbf{z}_{2}\right\|_{\mathbf{V}^{3 / 2-\varepsilon, 3 / 4-\varepsilon / 2}\left(Q_{\infty}\right)},
\end{aligned}
$$

for all $\mathbf{z}, \mathbf{z}_{1}, \mathbf{z}_{2} \in \mathbf{V}^{3 / 2-\varepsilon, 3 / 4-\varepsilon / 2}\left(Q_{\infty}\right)$. After that we study the mapping

$$
\Psi: \mathbf{z} \longmapsto \mathbf{y}_{\mathbf{z}},
$$

where $\mathbf{y}_{\mathbf{z}}$ is the solution to the system

$$
\begin{array}{ll}
P \mathbf{y}^{\prime}=A_{\Pi} P \mathbf{y}+F(\mathbf{z}) \quad \text { in }(0, \infty), & P \mathbf{y}(0)=\mathbf{y}_{0} \\
(I-P) \mathbf{y}=-(I-P) D_{A} R_{A}^{-1} B_{n}^{*} \Pi P \mathbf{y} & \text { in }(0, \infty) .
\end{array}
$$

To show that the mapping $\Psi$ is a contraction in $D_{\mu}$ (is $\mu_{0}$ and $\eta$ are suitably chosen), we prove the following Lemma. 
Lemma 1. ([15,Lemma 6.2]) For all $\mathbf{y}_{0} \in \mathbf{V}_{n}^{1 / 2-\varepsilon}(\Omega)$ and all $\mathbf{f} \in L^{2 /(1+2 \varepsilon)}\left(0, \infty ; \mathbf{V}_{n}^{0}(\Omega)\right) \cap L^{2}(0, \infty$; $\left.\left(\mathbf{V}^{2 \varepsilon}(\Omega)\right)^{\prime}\right)$ the solution to the equation

$$
\mathbf{y}^{\prime}=A_{\Pi} \mathbf{y}+\mathbf{f}, \quad \mathbf{y}(0)=\mathbf{y}_{0},
$$

obeys

$$
\|\mathbf{y}\|_{\mathbf{V}^{3 / 2-\varepsilon, 3 / 4-\varepsilon / 2}\left(Q_{\infty}\right)} \leq C_{1}\left(\left|\mathbf{y}_{0}\right|_{\mathbf{V}_{n}^{1 / 2-\varepsilon}(\Omega)}+\|\mathbf{f}\|_{L^{2 /(1+2 \varepsilon)}\left(0, \infty ; \mathbf{V}_{n}^{0}(\Omega)\right)}+\|\mathbf{f}\|_{L^{2}\left(0, \infty ;\left(\mathbf{V}^{2 \varepsilon}(\Omega)\right)^{\prime}\right)}\right) .
$$

After that, Theorem 5 follows from a fixed point argument.

\subsection{Second stabilization result}

To obtain a feedback law providing an exponential stabilization of the Navier-Stokes, we are going to use the linear feedback law determined thanks to an auxiliary problem. For that, we set

$$
\hat{\mathbf{y}}=e^{\omega t} \mathbf{y}, \quad \hat{\mathbf{u}}=e^{\omega t} \mathbf{u} \quad \text { with } \omega>0 .
$$

If

$$
\begin{aligned}
& P \mathbf{y}^{\prime}=A P \mathbf{y}+P F(\mathbf{y})+B \mathbf{u}, \quad P \mathbf{y}(0)=\mathbf{y}_{0}, \\
& (I-P) \mathbf{y}=(I-P) D_{A} \gamma_{\mathbf{u}},
\end{aligned}
$$

then $\hat{\mathbf{y}}$ is the solution to the system

$$
\begin{aligned}
& P \hat{\mathbf{y}}^{\prime}=A P \hat{\mathbf{y}}+\omega \hat{\mathbf{y}}+e^{-\omega t} F(\hat{\mathbf{y}})+B \hat{\mathbf{u}}, \quad P \hat{\mathbf{y}}(0)=\mathbf{y}_{0}, \\
& (I-P) \hat{\mathbf{y}}=(I-P) D_{A} \gamma_{n} \hat{\mathbf{u}} .
\end{aligned}
$$

Set $A_{\omega}=A+\omega I$, and let $\Pi_{\omega}$ be the solution to the algebraic Riccati equation:

$$
\Pi_{\omega}=\Pi_{\omega}^{*} \geq 0, \quad \Pi_{\omega} A_{\omega}+A_{\omega}^{*} \Pi_{\omega}-\Pi_{\omega} B_{\tau} B_{\tau}^{*} \Pi_{\omega}-\Pi_{\omega} B_{n} R_{A}^{-1} B_{n}^{*} \Pi_{\omega}+I=0 .
$$

The existence of a unique solution to this equation may be proved as in section 4 . Consider the Navier-Stokes equations with the linear feedback law:

$$
\begin{aligned}
& P \hat{\mathbf{y}}^{\prime}=A_{\Pi_{\omega}} P \hat{\mathbf{y}}+e^{-\omega t} F(\hat{\mathbf{y}}), \quad P \hat{\mathbf{y}}(0)=\mathbf{y}_{0}, \\
& (I-P) \hat{\mathbf{y}}=-(I-P) D_{A} R_{A}^{-1} B_{n}^{*} \Pi_{\omega} P \hat{\mathbf{y}},
\end{aligned}
$$

where

$$
A_{\Pi_{\omega}}=A+\omega I-B_{\tau} B_{\tau}^{*} \Pi_{\omega}-B_{n} R_{A}^{-1} B_{n}^{*} \Pi_{\omega} .
$$

As previously, if $\hat{\mathbf{y}}$ is a solution to (5.3), then $\mathbf{y}=e^{-\omega t} \hat{\mathbf{y}}$ is the solution of

$$
\begin{aligned}
& P \mathbf{y}^{\prime}=A P \mathbf{y}-B_{\tau} B_{\tau}^{*} \Pi_{\omega} P \mathbf{y}-B_{n} R_{A}^{-1} B_{n}^{*} \Pi_{\omega} P \mathbf{y}+F(\mathbf{y}), \quad P \mathbf{y}(0)=\mathbf{y}_{0}, \\
& (I-P) \mathbf{y}=-(I-P) D_{A} R_{A}^{-1} B_{n}^{*} \Pi_{\omega} P \mathbf{y} .
\end{aligned}
$$

Theorem 6. For all $0<\varepsilon<1 / 4$, there exists $\mu_{0}>0$ and a nondecreasing function $\eta_{0}$ from $\mathbb{R}^{+}$ into itself, such that if $\mu \in\left(0, \mu_{0}\right)$ and $\left\|\mathbf{y}_{0}\right\|_{\mathbf{V}_{n}^{1 / 2-\varepsilon}(\Omega)} \leq \eta_{0}(\mu)$, the equation (5.4) admits a unique solution in the set

$$
D_{\mu}=\left\{\mathbf{y} \in \mathbf{V}^{3 / 2-\varepsilon, 3 / 4-\varepsilon / 2}\left(Q_{\infty}\right) \mid\left\|e^{\omega(\cdot)} \mathbf{y}\right\|_{\mathbf{V}^{3 / 2-\varepsilon, 3 / 4-\varepsilon / 2}\left(Q_{\infty}\right)} \leq \mu\right\} .
$$

Moreover $\mathbf{y}$ belongs to $C_{b}\left([0, \infty) ; \mathbf{V}^{1 / 2-\varepsilon}(\Omega)\right)$, and it satisfies

$$
|\mathbf{y}(t)|_{\mathbf{V}^{1 / 2-\varepsilon}(\Omega)} \leq C(\mathbf{w}, \mu) e^{-\omega t} .
$$




\section{Stabilization of the three dimensional Navier-Stokes equations}

Throughout this section, we assume that $N=3$, w belongs to $\mathbf{V}^{5}(\Omega)$, and $\Omega$ is of class $C^{6}$. Let us first explain why the feedback law determined in section 4 is not convenient to stabilize the Navier-Stokes equations in 3 dimension.

In 3 dimension the mapping

$$
\mathbf{z} \longmapsto F(\mathbf{z})
$$

is locally Lispschitz from $\mathbf{V}^{3 / 2-\varepsilon, 3 / 4-\varepsilon / 2}\left(Q_{\infty}\right)$ into $L^{2}\left(0, \infty ;\left(\mathbf{V}^{1 / 2+2 \varepsilon}\left(Q_{\infty}\right)\right)^{\prime}\right)$. But we cannot prove that the mapping

$$
\mathbf{f} \longmapsto \int_{0}^{t} e^{(t-\tau) A_{\Pi}} \mathbf{f}(\tau) d \tau,
$$

is continuous from $L^{2}\left(0, \infty ;\left(\mathbf{V}^{1 / 2+2 \varepsilon}(\Omega)\right)^{\prime}\right)$ into $\mathbf{V}^{3 / 2-\varepsilon, 3 / 4-\varepsilon / 2}\left(Q_{\infty}\right)$, and we cannot use a fixed point argument as in 2 dimension. To deal with the nonlinear term $F(\mathbf{y})$ in 3 dimension, we have to look for solutions to the Linear-Quadratic problem in $\mathbf{V}^{3 / 2+\varepsilon, 3 / 4+\varepsilon / 2}\left(Q_{\infty}\right)$ for some $\varepsilon>0$ (or in $L^{2}\left(0, \infty ; \mathbf{V}^{3 / 2+\varepsilon}(\Omega)\right) \cap L^{\infty}\left(0, \infty ; \mathbf{V}^{1 / 2+\varepsilon}(\Omega)\right)$.

To obtain such a regularity result and to obtain a feedback law stabilizing the Navier-Stokes equation, an alternative way may be to choose the functional $I$ in problem $\left(\mathscr{P}_{\zeta}^{\infty}\right)$ so that the mapping

$$
\zeta \longmapsto\left(\Pi \mathbf{y}_{\zeta}(t), \mathbf{y}_{\zeta}(t)\right)_{\mathbf{V}_{n}^{0}(\Omega)},
$$

be a Lyapunov function of the closed loop system in $\mathbf{V}^{1 / 2+\varepsilon}(\Omega)$. This method has been successfully applied in the case of an internal control [1]. Its extension to the case of a boundary control (when the normal component of the control is equal to zero, and when the control is applied everywhere on the boundary $\Gamma$ ) has been studied very recently in [4]. The idea in [4] consists in choosing a functional $I$ coervive in $L^{2}\left(0, \infty ; \mathbf{V}^{3 / 2+\varepsilon}(\Omega)\right)$ for some $\varepsilon>0$. The $L^{2}\left(0, \infty ; \mathbf{V}^{3 / 2+\varepsilon}(\Omega)\right)$-regularity combined with the state equation of the Linear-Quadratic problem is sufficient to deal with the nonlinear term $F(\mathbf{y})$ in 3 dimension.

The price to pay is that the corresponding algebraic Riccati equation is only defined in $D\left(A_{\Pi}\right)$, which is itself unknown since $\Pi$ is not determined by an equation independent of $D\left(A_{\Pi}\right)$.

Here we follow a different approach. We modify the control problem $\left(\mathscr{P}_{\zeta}^{\infty}\right)$ in two ways. We modify both the cost function and the control operator. The control operator $B$ is replaced by $\theta B$, where the weight function $\theta \in C^{\infty}([0, \infty))$ satisfies

$$
\begin{aligned}
& \theta(t) \in[0,1] \quad \text { for all } t \in \mathbb{R}^{+}, \\
& \theta(0)=0 \quad \text { and } \quad \theta(t)=1 \quad \text { for all } t \geq T,
\end{aligned}
$$

with $T>0$ is given fixed. The new state equation is

$$
P \mathbf{y}^{\prime}=A P \mathbf{y}+\theta B \mathbf{u} \quad \text { in }(0, \infty), \quad \mathbf{y}(0)=\mathbf{y}_{0}, \quad \text { and } \quad(I-P) \mathbf{y}=(I-P) D_{A} \mathbf{u} .
$$

To understand the role of the weight function $\theta$, we observe that if $\mathbf{y}_{0} \in \mathbf{V}_{0}^{1 / 2+\varepsilon}(\Omega)$ and if $\mathbf{u} \in$ $\mathbf{V}^{1+\varepsilon^{\prime}, 1 / 2+\varepsilon^{\prime} / 2}\left(\Sigma_{\infty}\right)$ for some $\varepsilon^{\prime}>\varepsilon>0$, then the solution to equation (6.1) belongs to $L_{\text {loc }}^{2}([0, \infty)$; $\left.\mathbf{V}^{3 / 2+\varepsilon}(\Omega)\right) \cap H_{\text {loc }}^{3 / 4+\varepsilon / 2}\left([0, \infty) ; \mathbf{V}^{0}(\Omega)\right)$. This regularity result is obtained because the trace of the initial condition $\mathbf{y}_{0}$ and the initial value of the boundary control $\theta \mathbf{u}$ are both equal to zero, and 
consequently they satisfy a compatibility condition which cannot be obtained without the presence of $\theta$.

In the cost function we replace $\int_{0}^{\infty} \int_{\Omega}|P \mathbf{y}|^{2}$ by $\int_{0}^{\infty} \int_{\Omega}\left|\left(-A_{0}\right)^{-1 / 2} P \mathbf{y}\right|^{2}$. More precisely we consider the family of control problems

$\left(\mathscr{Q}_{\bar{t}, \mathbf{y}_{0}}\right) \quad \inf \left\{I(\bar{t}, \mathbf{y}, \mathbf{u}) \mid(\mathbf{y}, \mathbf{u})\right.$ satisfies $\left.(6.2), \mathbf{u} \in \mathbf{V}^{0,0}\left(\Sigma_{\bar{t}, \infty}\right)\right\}$,

where

$$
I(\bar{t}, \mathbf{y}, \mathbf{u})=\frac{1}{2} \int_{\bar{t}}^{\infty} \int_{\Omega}\left|\left(-A_{0}\right)^{-1 / 2} P \mathbf{y}\right|^{2}+\frac{1}{2} \int_{\bar{t}}^{\infty} \int_{\Gamma}\left|R_{A}^{1 / 2} \gamma_{n} \mathbf{u}\right|^{2}+\frac{1}{2} \int_{\bar{t}}^{\infty} \int_{\Gamma}\left|\gamma_{\tau} \mathbf{u}\right|^{2}
$$

and

$$
P \mathbf{y}^{\prime}=A P \mathbf{y}+\theta B \mathbf{u} \quad \text { in }(\bar{t}, \infty), \quad P \mathbf{y}(\bar{t})=\mathbf{y}_{0}, \quad \text { and } \quad(I-P) \mathbf{y}=(I-P) D_{A} \mathbf{u} .
$$

Thus contrarily to what is done in [1] and in [4], where a norm stronger than the $L^{2}$-norm is used in the cost function, here we take a norm weaker than the $L^{2}$-norm. The regularity result for the optimal state is recovered by studying the optimality system.

Theorem 7. For all $\mathbf{y}_{0} \in \mathbf{V}_{n}^{0}(\Omega)$ and all $\bar{t} \in[0, \infty)$, problem $\left(\mathscr{Q}_{\bar{t}, \mathbf{y}_{0}}\right)$ admits a unique solution $\left(\mathbf{y}_{\bar{t}, \mathbf{y}_{0}}, \mathbf{u}_{\bar{t}, \mathbf{y}_{0}}\right)$. There exists $\Pi(\bar{t}) \in \mathscr{L}\left(\mathbf{V}_{n}^{0}(\Omega)\right)$ such that the optimal cost is given by

$$
I\left(\bar{t}, \mathbf{y}_{\bar{t}, \mathbf{y}_{0}}, \mathbf{u}_{\bar{t}, \mathbf{y}_{0}}\right)=\frac{1}{2}\left(\Pi(\bar{t}) \mathbf{y}_{0}, \mathbf{y}_{0}\right)_{\mathbf{V}_{n}^{0}(\Omega)} .
$$

Proof. The proof is completely analogous to the one in [15, Theorem 4.1] or to the proof of Theorem 2.

Lemma 2. (see [16]) For every $\mathbf{y}_{0} \in \mathbf{V}_{n}^{0}(\Omega)$, the system

$$
\begin{aligned}
& P \mathbf{y}^{\prime}=A P \mathbf{y}-\theta^{2} B_{\tau} B_{\tau}^{*} \Phi-\theta^{2} B_{n} R_{A}^{-1} B_{n}^{*} \Phi \quad \text { in }(\bar{t}, \infty), \quad \mathbf{y}(\bar{t})=\mathbf{y}_{0}, \\
& -\Phi^{\prime}=A^{*} \Phi+\left(-A_{0}\right)^{-1} P \mathbf{y} \quad \text { in }(\bar{t}, \infty), \quad \Phi(\infty)=0, \\
& \Phi(t)=\Pi(t) P \mathbf{y}(t) \quad \text { for all } t \in(\bar{t}, \infty), \\
& (I-P) \mathbf{y}(t)=-(I-P) D_{A} \theta^{2} B_{n} R_{A}^{-1} B_{n}^{*} \Pi(t) P \mathbf{y}(t) \quad \text { in }(\bar{t}, \infty)
\end{aligned}
$$

admits a unique solution in $L^{2}\left(\bar{t}, \infty ; \mathbf{V}_{n}^{0}(\Omega)\right) \times \mathbf{V}^{2,1}\left(Q_{\bar{t}, \infty}\right)$. This solution belongs to $C_{b}\left([\bar{t}, \infty) ; \mathbf{V}_{n}^{0}(\Omega)\right)$ $\cap \mathbf{V}^{1,1 / 2}\left(Q_{\bar{t}, \infty}\right) \times\left(L^{2}\left(\bar{t}, \infty ; \mathbf{V}^{5}(\Omega)\right) \cap H^{3 / 2}\left(\bar{t}, \infty ; \mathbf{V}^{2}(\Omega)\right)\right)$ and it satisfies:

$$
\|\mathbf{y}\|_{C_{b}\left([\bar{t}, \infty) ; \mathbf{V}_{n}^{0}(\Omega)\right)}+\|\mathbf{y}\|_{\mathbf{V}^{1,1 / 2}\left(Q_{\bar{t}, \infty}\right)}+\|\Phi\|_{L^{2}\left(\bar{t}, \infty ; \mathbf{V}^{5}(\Omega)\right) \cap H^{3 / 2}\left(\bar{t}, \infty ; \mathbf{V}^{2}(\Omega)\right)} \leq C\left|\mathbf{y}_{0}\right|_{\mathbf{V}_{n}^{0}(\Omega)} .
$$

The pair $\left(\mathbf{y},-\theta B_{\tau}^{*} \Phi-\theta R_{A}^{-1} B_{n}^{*} \Phi\right)$ is the solution of $\left(\mathscr{Q}_{\bar{t}, \mathbf{y}_{0}}\right)$.

Corollary 2. (see [16]) If $\mathbf{y}_{0} \in \mathbf{V}_{n}^{1 / 2-\varepsilon}(\Omega)$ for some $0<\varepsilon<1 / 2$, then the solution $(\mathbf{y}, \Phi)$ of system (6.3) belongs to $\mathbf{V}^{3 / 2-\varepsilon, 3 / 4-\varepsilon / 2}\left(Q_{\bar{t}, \infty}\right) \times\left(L^{2}\left(\bar{t}, \infty ; \mathbf{V}^{11 / 2-\varepsilon}(\Omega)\right) \cap H^{7 / 4-\varepsilon / 2}\left(\bar{t}, \infty ; \mathbf{V}^{2}(\Omega)\right)\right)$, and we have:

$$
\begin{aligned}
& \|\mathbf{y}\|_{\mathbf{V}^{3 / 2-\varepsilon, 3 / 4-\varepsilon / 2}\left(Q_{\bar{t}, \infty}\right)}+\|\Phi\|_{L^{2}\left(\bar{t}, \infty ; \mathbf{V}^{11 / 2-\varepsilon}(\Omega)\right) \cap H^{7 / 4-\varepsilon / 2\left(\bar{t}, \infty ; \mathbf{V}^{2}(\Omega)\right)}} \leq C\left|\mathbf{y}_{0}\right|_{\mathbf{V}_{n}^{1 / 2-\varepsilon}(\Omega)}, \\
& \left\|B^{*} \Phi\right\|_{L^{2}\left(\bar{t}, \infty ; \mathbf{V}^{4-\varepsilon}(\Gamma)\right) \cap H^{3 / 4-\varepsilon / 2}\left(\bar{t}, \infty ; \mathbf{V}^{5 / 2}(\Gamma)\right)} \leq C\left|\mathbf{y}_{0}\right|_{\mathbf{V}_{n}^{1 / 2-\varepsilon}(\Omega)} .
\end{aligned}
$$


If $\mathbf{y}_{0} \in \mathbf{V}_{0}^{1 / 2+\varepsilon}(\Omega)$ for some $0 \leq \varepsilon \leq 1 / 2$ and if $\bar{t}=0$, then the solution $(\mathbf{y}, \Phi)$ of system (6.3) belongs to $\mathbf{V}^{3 / 2+\varepsilon, 3 / 4+\varepsilon / 2}\left(Q_{\infty}\right) \times\left(L^{2}\left(0, \infty ; \mathbf{V}^{11 / 2+\varepsilon}(\Omega)\right) \cap H^{7 / 4+\varepsilon / 2}\left(0, \infty ; \mathbf{V}^{2}(\Omega)\right)\right)$, and we have:

$$
\|\mathbf{y}\|_{\mathbf{V}^{3 / 2+\varepsilon, 3 / 4+\varepsilon / 2}\left(Q_{\infty}\right)}+\|\Phi\|_{L^{2}\left(0, \infty ; \mathbf{V}^{11 / 2+\varepsilon}(\Omega)\right) \cap H^{7 / 4+\varepsilon / 2}\left(0, \infty ; \mathbf{V}^{2}(\Omega)\right)} \leq C\left|\mathbf{y}_{0}\right|_{\mathbf{V}_{n}^{1 / 2+\varepsilon}(\Omega)},
$$

and

$$
\left\|B^{*} \Phi\right\|_{L^{2}\left(0, \infty ; \mathbf{V}^{7 / 2+\varepsilon}(\Omega)\right) \cap H^{3 / 4+\varepsilon / 2}\left(0, \infty ; \mathbf{V}^{2}(\Omega)\right)} \leq C\left|\mathbf{y}_{0}\right|_{\mathbf{V}_{n}^{1 / 2+\varepsilon}(\Omega)} .
$$

Due to this regularity result we are able to define a feedback law which stabilizes the Navier-Stokes equations (see [16]).

\section{References}

[1] V. Barbu, Feedback stabilization of the Navier-Stokes equations, ESAIM COCV, 9 (2003), 197-206.

[2] V. Barbu, S. S. Sritharan, $H^{\infty}$ - control theory of fluid dynamics, Proc. R. Soc. Lond. A 454 (1998), 3009-3033.

[3] V. Barbu, R. Triggiani, Internal stabilization of Navier-Stokes equations with finite dimensional controllers, Indiana University Journal, 52(5) (2004), 1443-1494.

[4] V. Barbu, I. Lasiecka, R. Triggiani, Boundary stabilization of Navier-Stokes equations, Memoirs of the A.M.S., 2005, to appear.

[5] E. Fernandez-Cara, S. Guerrero, O. Yu. Imanuvilov, J.-P. Puel, Local exact controllability of the Navier-Stokes system, 83 2004, 1501-1551.

[6] A. V. Fursikov, M. D. Gunzburger, L. S. Hou, Trace theorems for three-dimensional time-dependent solenoidal vector fields and their applications, Transactions of the American Mathematical Society, Vol. 354 (2001), 1079-1116.

[7] A. V. Fursikov, M. D. Gunzburger, L. S. Hou, Inhomogeneous boundary value problems for the three-dimensional evolutionary Navier-Stokes equations, J. Math. Fluid Mech., 4 (2002), 45-75.

[8] A. V. Fursikov, Stabilization for the 3D Navier-Stokes system by feedback boundary control, Discrete and Cont. Dyn. Systems, 10 (2004), 289-314.

[9] G. Grubb, Nonhomogeneous Dirichlet Navier-Stokes problems in low regularity $L_{p}$ Sobolev spaces, J. Math. Fluid Mech., Vol. 3 (2001), 57-81.

[10] B. B. King, Y.-R. Ou, Nonlinear dynamic compensator design for flow control in a driven cavity, Proc. 34th CDC, Dec. 1995, 3741-3746.

[11] J.-W. He, R. Glowinski, R. Metcalfe, A. Nordlander, J. Periaux, Active control and drag optimization for flow past a circular cylinder, JCP 163 (2000), 83-117.

[12] J.-L. Lions, E. Magenes, Problèmes aux limites non homogènes, Vol. 2, Dunod, Paris 1968.

[13] D. S. Park, D. M. Ladd, and E. W. Hendricks, Feedback control of von Karman vortex shedding behind a circular cylinder at low Reynolds numbers, Phys. Fluids, 6 (1994), 2390-2405.

[14] J.-P. Raymond, Stokes and Navier-Stokes equations with nonhomogeneous boundary conditions, 2005, submitted.

[15] J.-P. Raymond, Boundary feedback stabilization of the two dimensional Navier-Stokes equations, 2005, submitted. 
[16] J.-P. Raymond, Boundary feedback stabilization of the three dimensional Navier-Stokes equations, in preparation.

[17] R. Temam, Navier-Stokes equations, North-Holland, 1984. 